\title{
Learning Algorithms, Design, and Computed Space
}

\author{
Roberto Bottazzi
}

\begin{abstract}
The paper analyses and speculates on what opportunities and challenges will arise from the introduction of learning algorithms (machine learning, neural networks, etc.) in architectural and urban design. The penetration of such class of algorithms in cities and design disciplines is rapid and profound, increasing both the thirst for gathering ever larger and more accurate datasets and raising the prospect of automating tasks currently performed by humans. Whilst it is understood that learning algorithms are essential tools to analyse large datasets, design disciplines have paid far less attention to how such processes are carried out, how spatial data are reformatted by algorithms which largely operate on statistical bases and, most importantly, what image of the city emerges from such processes. To unravel the complexity of the issue, it is first necessary to retrace the ideas informing the emergence of numerical procedures as they developed since the beginning of the twentieth century as they allow us to project a different paradigm of how space can be analysed, structured, and changed. Finally, the paper will offer some points for speculation and further reflection on how the methods put forward through learning algorithms compare to current approaches to digital design; this will foreground their disruptive potential for a radical transformation of urban design, one that could be deployed to tackle some of the most pressing urban issue.
\end{abstract}

Keywords: Artificial Intelligence, Big Data, Urban Design

\section{Introduction}

Over the past decade a new breed of algorithms has rapidly spread through society, impacting almost all aspects including cities and urban design. Though these algorithmic processes are not native to spatial design disciplines, as they belong to a particular subbranch of computer sciences dedicated to the development of artificial intelligence, their impact on design is proving to be profound. Throughout this paper, we will refer to them as Learning Algorithms (LA) and position them at a junction between the latest developments in Artificial Intelligence (AI) and
Big Data. Thanks to the type of computational architecture structuring them, LA perform a type of "learning" that radically differs from the human brain as their basis for learning is solely provided by on the datasets they are fed with. Different from more established programming paradigms, they have not been programmed by explicitly pre-determining all the steps nor by assigning fixed values to parameters. Rather, LA are iterative procedures - sometime indicated as convolution - which adjust variables to self-adapt (what is generally understood as learning) to improve their performativity to achieve or

Stable URL: https://arcc-journal.org/index.php/arccjournal/article/view/1058 10.17831/enq:arcc.v16i1.1058

Corresponding Author: roberto.bottazzi@ucl.ac.uk

Published by the Architectural Research Centers Consortium under the terms of the Attribution-NonCommercial-ShareAlike 4.0 International license 
approximate a target. Such target can either be predetermined (supervised learning) or emerging (unsupervised learning). As we will see in greater depth, their historical origin is situated in the field of computational studies for the development of $\mathrm{Al}$ which started in the 1950s and are finding extensive applications in the analysis of massive datasets as we normally encounter in the age of Big Data. LA are an integral part of Big Data as they have become the best, and sometime only, method available to mine colossal sets of data. ${ }^{1}$ The design experiments accompanying this discussion have largely employed supervised learning methods and combine a series of procedures including: Principal Components Analysis (PCA), t-Distributed Stochastic Neighbor Embedding (t-SNE), Neural Networks (supervised and unsupervised).

Whilst there has been considerable technical advancements and widespread applications (to routine tasks on smartphones, regulate financial transactions, estimate risk for insurance companies, etc.), their application to spatial disciplines is still in an embryonic state and even more primitive are theoretical and historical reflections on what they could bring to such disciplines. This paper can be seen as a preliminary series of considerations on the role of LA based on a series of design carried out at The Bartlett School of Architecture at UCL.

The approach to this set of related issues taken here is to first deepen our understanding of how LA work to better critique how they differ from other computational approaches to design and be able to speculate how they might impact on urban design. This process begins by first grasping the genealogy of LA through which will foreground a different model of distribution at work and, consequently, of spatial organisation. A critical appraisal of the history of such technical models will return an image of spatial computation which we will argue is at odds with the direction and terminology that digital designers have brandished over the past decades. Not only will it be useful to point out these moments of crisis, but also to do so by coupling theoretical discussions with examples of practical applications. The design examples illustrating this discussion come from the work developed by Research Cluster 14 (RC14) part of the Master in Urban Design at The Bartlett School of Architecture at UCL which I lead with Dr. Tasos Varoudis.

Underpinning the whole discussion is the aim to show that computational methods adopting LA offer more than often-celebrated optimised efficiency of algorithmic procedures. The complexity and richness of operations performed allows us to also discuss LA in terms that deviate the conversation away from pure efficiency in, at least, two ways. First, LA operate iteratively by producing colossal number of options to solve a problem. Though only a fraction of these may worthy of further speculation, the whole process can offer new perspectives on a given problem, therefore widening the range of the spectrum analysed. The possibility to handle such large numbers of options allows to scope out all the design space. Such possibility is one way in which the inhuman nature of computation becomes intelligible prompting philosophers to speak of computation as "alien". That is, algorithms may be "thinking" but not in ways we can follow. This issue can also be read in terms of aesthetics as exploiting LA and their inhuman nature often also involves confronting uncanny images. Artistic work to capture the potential of Al aesthetic represents one of the most interesting developments in creative disciplines.

One of the first moments of crisis we encounter has thus do to with the narrative frames utilised to understand the role of algorithms in the design process. One of the most durable metaphors for CAD is the image of the "perfect slave" (Coons 1966) coined by Coons at MIT. It is however hard to cast LA as mere, somehow "inferior" helpers in the design process, especially when they can easily operate with datasets that exceed human cognition by many orders of magnitude. This is no longer just a technical feature, but an epistemological issue that cannot be brushed aside through the master-slave metaphor. On the contrary, designers will need to tune their imaginations to very nature of how learning algorithms operate or even how algorithms interact with each other. This will require new type of computational literacy; a task we propose to develop
${ }^{1}$ The Wikipedia definition of Big Data describes it as “...a field that treats ways to analyze, systematically extract information from, or deal with data sets that are too large or complex to be dealt with by traditional data- processing application software. From https://en.wikipedia.org/wiki/Big_data [last accessed on 15th June, 2019]. 
by moving back and forth between historical and contemporary concepts and techniques to organise space. What would it mean to manage and design cities in these mutated conditions? What will the full effect of deploying principles mutated from artificial intelligence be on design? And, most crucially, what kind of spatiality can learning algorithms engender?

\section{Early steps towards the development of Learning Algorithms: Dartmouth College and Markov's experiments.}

As part of the development of Al, learning algorithms were already of concern to computer scientists in the 1950 s as the work Alan Turing evidences (Turing 1950). However, the first official definition of Al was provided in 1955 when J. McCarty, M. Minsky, N. Rochester, and C. Shannon proposed a summer workshop at Dartmouth College (McCarthy, et al 1955). The definition of Al put forward at Dartmouth was consistent with Turing's experiment - now better known as "Turing Test" - and posited that "...artificial intelligence is taken to be that of making a machine behave in ways that would be called intelligent if a human were so behaving" (ibid.). This is, the emphasis was on the outcome (the machine can pass as a human), and not on whether the thinking process followed or bore any resemblance to the neural workings of the brain; in other words, from the outset, Al privileged the performative rather the cognitive approach to emulating intelligence. Such results heavily relied on numerical approaches which suggested the possibility to generate "intelligent" behaviour not modelled on human intelligence. Applied to data, this consideration meant that it could have bene possible to extract information from datasets without having any traditional insight or knowledge of the material analysed. This approach is still at the core of how learning algorithms correlate data and adapt themselves to better their performance without any semantic understanding of the content manipulated. An earlier and very powerful example of such kind of work is the experiment carried out by Andrei Markov in 1913. Markov took a text written by Pushkin and sampled it into blocks of 100 letters each. He then proceeded to count the letters' distribution and statistically extracted patterns (e.g. number and position of vowels and consonants) and statistical deviances which allowed him to operate the first mathematical treatment of language (Link 2016, pp. 29-54).

Markov's algorithm learnt something about Pushkin's text by purely recoding it into numbers with no semantic or narrative content. It did so by circumventing traditional methods to focus on the relational qualities of the dataset. Spatial distribution became an indicator of order, whereas statistical probability replaced linearity and strict causal relations. Both steps are important not only because they begin to show how numerical procedures may operate, but also because LA could be describe a similar processes which are performed at a massive scale on a much more intricate network of iterative connections. However, the modes in which an algorithm apprehends a dataset do have their properties and constrains which also offers models to rethink the relation between computation and design.

\section{Algorithms and space}

Before delving into the specific procedures of LA, we ought to draw some general considerations on the interaction between data, algorithms, and space. As we narrow the discussion to design, we should foreground what LA can bring in order to forge new relations between data and space. LA not only dictates the rules of engagements between data, space, and products, but also determines the very bounds of the design space. ${ }^{2}$ Lev Manovich identified in software the locus of this relation as it both engenders and constrains creative ideas; a frame of reference to build upon in this discussion (Manovich 2013). However in the case of $L A$, it is perhaps more apt to take a step back and speak of code rather than software. Formed by a mix of language, numbers, and mathematical symbols, code connects data and design providing the virtual space in which techniques can be charged with conceptual intentions and agency. How do algorithms apprehend datasets, especially if these are spatial? What new elements do LA introduce in the design process? What kind new spatiality they engender?

As mentioned, Markov's experiment already offers hints on how numerical models can see and structure

2 In Alejandro Zaera-Polo's words: "nothing gets built that isn't transposed onto AutoDesk AutoCAD." 
space (albeit Markov only explored the space of the text). Such model is solely organised by mathematics and carries the possibility to be automated through the introduction of a machine. What is at stake here is how the algorithm generates a new image and possibly a different kind of knowledge out of an initial dataset. When Carlo Sini approached similar issues from the point of view of philosophy, he felt compelled to turn to Nietzsche's own definition of knowledge to frame and generalise how to think about algorithmic processes. Nietzsche defined knowledge as the process of moving something from the domain of the unknown or stranger into that of the known or familiar (Nietzsche 1975 cited in Sini 2009 , p.48). This definition could stand its application to the operation of LA: their application to an 'unknown' object opens up a complex process to make such object intelligible. However, as Nietzsche also warned, the aim should not be to make the final result familiar, which would terminate the process of learning, but rather to improve its intelligibility by highlighting aspects of it, by realising the artificiality of the whole process, and, ultimately, by foregrounding the incompleteness of the process of learning. Sini extended these considerations by pointing out that knowledge is a form of "labour" on the "unknown" and, therefore, the feedback between action and knowledge cannot ever exhaust or fully resolve each other. As such, the whole process reveals prosthetic qualities, here not understood according its contemporary, often medical meaning, but rather in its Greek origin (protithemi), that is, to present, exhibit, assign, etc. It is along these lines we can think that an algorithm applied to a dataset is doing some form of labour on it which will return a new image of it determined by its very algorithmic structure. This process is broadly two-fold: the algorithm first discriminates between what will be computed and what will not (epistemological threshold) and, then, re-writes a new image of the original dataset. Markov's procedure provided such image (intelligibility) by discretising the original data into individual letters, counting them (according to the rules of the algorithm) and making amenable to further analysis by applying statistical methods. Contemporary examples of algorithmic procedure allow us to apply and generalise some of these considerations to spatial datasets as in the case of the Marching Cube algorithm. ${ }^{3}$ This algorithm is used, amongst many examples, in medical imaging, to scan three-dimensional scalar numerical fields (generated from MRI scans) by iteratively analysing voxels comprising of eight numerical values (the vertex of the voxel space) at the time. Any numerical value below a pre-established threshold (called iso-value) is normalised to 0 , whereas to the opposite conditions is given the value of 1 (in our argumentation, this step represents the determination of an epistemological threshold). The new set of binary numbers are encoded following in one of the 256 codified cases determining the position and shape of a polygon; finally, the individual polygons are fused together in a mesh (new image). Following Sini's approach, these operations align the algorithm to a prosthesis of the dataset to which it is applied (Sini 2009, pp. 53-62). In this context, the term denotes two different activities: it indicates a process of "exteriorisation" of data and the act of measuring it. In the first operation, a new dataset is produced which distinguishes itself from the original and becomes amenable to further investigation - whether numerical or not. With LA particularly neural networks - this process can be very deep as it can run over a high number of layers to eventually become untraceable. This produces two further issues: one is theoretical and the other sociopolitical. The image of knowledge as an open process always necessitating work, whether performed by physical or immaterial tools, is only reinforced by the introduction of LA: stacking of several algorithms acting on different layers makes the issue of legibility more pressing as algorithms will perform some sort of work as long as syntactically possible. Consequently, scientists have warned against "spurious correlations" and called for a more holistic understanding of phenomena modelled through LA. ${ }^{4}$ From a social and political point of view, the insertion of ever more layers between initial dataset and final outputs creates a potentially massive gap between the set of data the process started with and the conclusions it ended up suggesting, an unbridgeable epistemological gulf. The case of Cambridge
3 Marching Cubes Algorithm. Available from: https://en.wikipedia.org/wiki/Marching_cubes [accessed on July 4th, 2019].
${ }^{4}$ Longo, G, Calude, C. S. (2017). "The Deluge of Spurious Correlations in Big Data". In Foundations of Science, 2017, Volume 22, Issue 3, pp. 595-612. 
Analytica ${ }^{5}$ is a point in case of this latter issue as the company was able to extrapolate deep and profound statistical correlations suggesting users' political orientations based on fairly generic and "innocent" input data (fashion preferences). To evidence such claims, it suffices to register the popularity of initiatives on the ethics of $\mathrm{Al}$, clearly signalling both the extents of this problem and inefficacy of traditional modes of thinking. Secondly, algorithms operate as basic "...analytical-descriptive 'unit of measure' of the world" (ibid., p. 55) implying that different types of algorithms will offer different 'units of measurements' and consequently give rise to different images. In Markov's work, such unit was strictly numerical and foregrounded a new image of Pushkin's novel to reveal discontinuities and patterns not evident in the original piece. Such act of measuring also withered the division between quantitative and qualitative aspects of data, therefore expanding numerical and logical operations to acquire design agency. This only shows the limited traction that comparing algorithms to "perfect slaves" has on LA. How LA see a dataset is different to humans and, as such, it cannot be thought of as a mere continuation of traditional activities but rather as a disruptive, 'alien' force. It is again Nietzsche who reminds us that the extraction of patterns from the flow of reality does not equate to knowledge yet, nor should there be any anxiety in fixing such flow as the process of discovery is an ongoing endeavour proceeding through approximations and loops (Nietzsche 1975, p.177). More enthusing is the possibility to task algorithms to perform similar actions on spatial databases to tease out certain, "alien" aspects and to probe them to initiate the complex and approximating process of knowledge acquisition. Along the same lines, we can also argue that computational approaches to design are best understood as methods to broaden spatial explorations, to surface novel arrangements, and to

${ }^{5}$ We are referring to the recent scandal that involved the Big Data company Cambridge Analytica. In brief, the company was accused of mining social media data in order to affect results of political elections or referenda. As revealed by Christopher Wylie - the whistle-blower that initiated the whole investigation - when hired to support Donald Trump's 2016 US presidential campaign, Cambridge Analytica algorithmically analysed patterns in social media profiles in search for the most robust indicators of individuals' political. Wylie's analysis identified fashion taste as the most search for different types of spatial organisations breaking free from the mantra of efficiency. Design epistemology through algorithmic design is better described as an act of labour characterised by a more complex, iterative idea of working with algorithms to approximate, refine, and, eventually, construct knowledge. Such process can take a variety of forms: by operating through substitutions, developing multiple experiments, testing options in order to develop a different vantage point through which to see the same dataset and, in the case of spatial data, aspects of cities; it is both a precise and open interaction that aligns itself more to the mechanisms of search for novelty and synthesis - two cornerstones of design - rather than efficiency and reductionism.

Of course, transferring models from the field of mathematics to design poses key problems which will warrant deeper analysis. Two issues worth mentioning in passing here have to do with the notion of data and that of time. The former has to do with the "double representation" of reality that data are: first through translating "the world" into numbers and then by further transforming them data. Data is the starting point of algorithmic operations, the risk of a naturalisation of data is of course implicit in this representational chain. The former concerns the temporal gap between data which are by definition static (some form of discretisation of reality) and the dynamic nature of urban environments. This gap, by definition, adds a further level of instability to this computational architecture of epistemology.

\section{Design with Learning Algorithms}

This long foray into algorithmic processes dissected from a technical, historical, and philosophical point of view shifts our discussion on LA and how they can impact spatial design.

reliable evidence of individual political biases. Ferrier, M. (2018). Christopher Wylie: 'The fashion industry was crucial to the election of Donald Trump', in The Guardian, November 29, 2018, Available at: https://www.theguardian.com/fashion/2018/nov/29/c hristopher-wylie-the-fashion-industry-was-crucial-tothe-election-of-donald-trump [accessed on June 22, 2019]. 
This framework also allows to apprehend earlier, predigital designs which conceptually align well with our considerations on LA. The work developed by Leonardo and Laura Mosso on the Piedmont rural landscape can be reassessed against the description of algorithmic processes previously outlined (Baccaglioni Del Canto 1981, pp. 26-8). Here the algorithm is physical rather than logical as it is represented by a network of springs - part of their long research on joints - which are overlaid on topographical maps of the Piedmont countryside. What may apparently be missing by not directly employing digital computers is gained in terms of clarity: we can literally see the two-fold prosthetic qualities of algorithms. The project speculates new types of organisation of the countryside by literally placing clusters of springs on physical models: these form new morphologies which are directly based on the information on the landscape recorded on the map. As prostheses, they make the landscape speak, albeit in a 'language' they set and control. Such language resides in their physical properties which control the process of "exteriorisation" - as mentioned by Sini - based on their length, type of connection, limiting the range of angles each member can take, and their collective behaviour. The outcome is, again, a new and different image of the rural hills of Piedmont, emerging from a process that allowed to re-write the original topography, opening up a new approach, and, potentially, speculating new ways to intervene.

More recently, Alisa Andrasek's Fissures (2012) exhibits an elegant use of a series of computational agents that distort and erode a datascape according to a set of coded instructions guiding their behaviour. ${ }^{6}$ The computational process which eventually provides the geometry for the main structure for the roof of a ferry terminal possesses the elements mentioned above including the capacity to play with inconsistencies and complexities arising from numerical calculations.

As we slowly zoom in on design work with direct use of $L A$, we notice in passing the radical impact that technologies of automation are having on society reconfiguring the relation between codes, machines, and space. As elaborated in greater detail elsewhere (Bottazzi 2018, pp. 13-38), received notions of scale, territory, representation and type quickly become outdated and replaced by the numerical space of data and algorithms. This can be seen through the emerging typology of distribution centres: these buildings are literal architectural computing machines as they are animated by robots rather than humans and strictly organised around the logic of algorithms. Their relevance is not aesthetic but organisational as they eventually impact cities by reformatting the way in which production and distribution work. ${ }^{7}$ Beyond metaphors of slavery, LA shapes a "nonproportional and electronic" (Hayles 1993) space in which the granular scale of the individual data point coexists with that of the planet. It is a disruptive force infiltrating cities rather than the pacifying image of efficiency, expanding the remit of design to encompass domains previously accessible. Data can represent scales that far exceed those tackled by designers and so can algorithms to engage it: gathered on a planetary level, with higher precision and potentially updated in real time, digital data allows to speculate beyond what customarily has been defined as the urban. In doing so, new models for spatial organisation can emerge.

First, as already implied in the initial considerations on prosthetic thinking, the computational approaches structured through LA enable the possibility of designing 'all at once' (Fig.1). We are not referring to the idea of data casting all-encompassing gaze, and, perhaps, control. Less emphatically and problematically, algorithms can analyse an entire dataset as well as carry out asynchronous or multiple computations on it.

Whereas other traditional analogue design methods segment complex processes into manageable tasks, LA apply their procedures to the entire dataset, therefore working with it in its original complexity avoiding, or at least limiting, reductions in either size or dimensionality. Even when data dimensionality does get reduced, as in the case of t-SNE algorithms ${ }^{8}$, it maintains its original qualities as we can still

7 Here Markov's influence is literal rather than metaphorical as Markov chains are still largely utilised on, for instance, websites to direct customers' choices.

8 t-distributed stochastically neighbour embedding. Available from: https://en.wikipedia.org/wiki/T-
${ }^{6}$ The full video illustrates the design process followed for the design of the roof of this completion entry for a ferry terminal. Biothing (2012). Fissures. [video] available at: https://vimeo.com/40401239_[accessed on 14th of June, 2019]. 


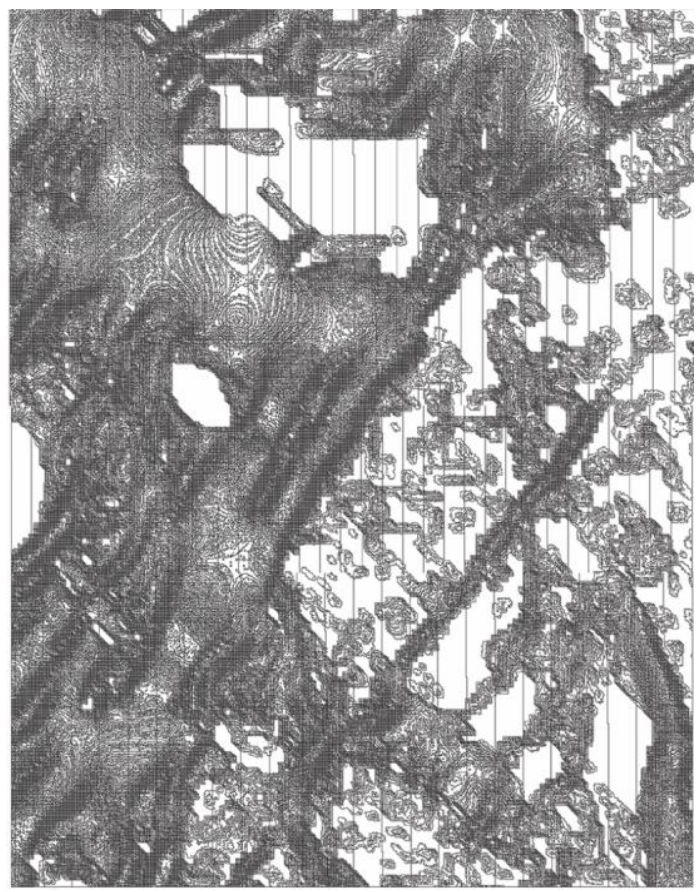

Figure 1: Principal Component Analysis of air pollution data identifying different clusters of risk and potential intervention. appreciate the disjunctions or continuities between the original dataset and the new image conjured up by the algorithm.

When applied to geo-located data, the disjunction between the spatial distribution of geo-located data and their algorithmic remapping in an abstract space shows more clearly the kind of spatial challenges this process present (Fig.2). What may have been continuous in the geographical representation (therefore identifying a homogenous zone) may not be in the abstract space of the algorithm and vice versa. Such metastable configurations are no longer a priori reducible to overarching or continuous spatial models: clustering of similar data can be punctual or extensive; distribution of patterns not only escapes linear hierarchies, but also becomes the expression of the abstract logic of digital computation. For this reason, the work developed within RC14 at The Bartlett extensively employs granular, discrete morphological languages that can take on more discontinuous organisational principles. Moreover, a fragmented design language more easily absorbs different dimensions of data as it can be layered with different qualities (material, colour, dynamic
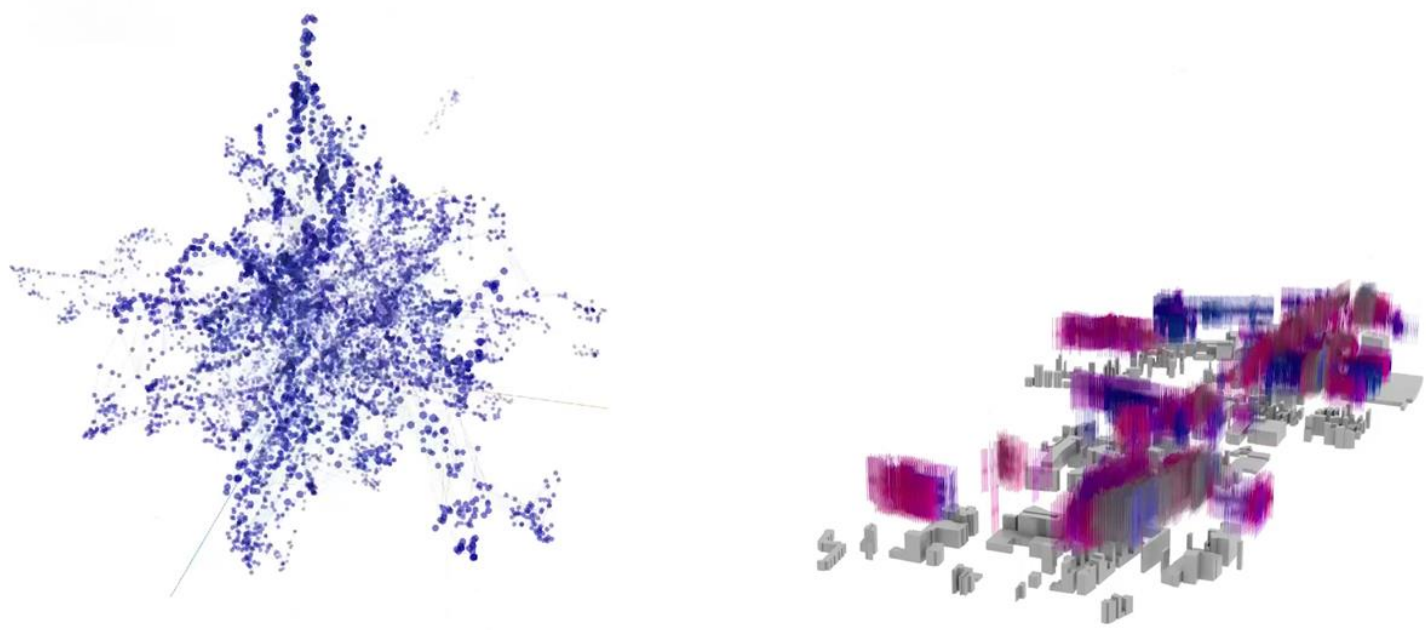

Figure 2: Then two images represent the same geo-located dataset in two different spaces. On the left, this is distributed in the abstract space of t-SNE algorithm, whereas on the right data is geo-located. LA propose a complex, discontinuous image of space in the city: data point which may be adjacent or clustered in the t-SNE visualisation could be distant in the map and vice versa. These exercises are key to realise the different spatiality suggested by LA and, therefore, instrumental in developing a design language and approach that can work with them.

distributed_stochastic_neighbor_embedding [accessed

on June 20th, 2019]. 
behaviour, etc.) and can branch out into several, potentially independent, systems. What can be gained is the possibility to absorb and exploit both the granular qualities of the individual data points and their relationality, continuously moving between the individual and the whole. The openness and potentially fragmented qualities of a discretised, almost quantum, design language mean that we do not need to relinquish the individual data point within the whole: the process of averaging that unavoidably takes place to spatially remap complex datasets in space can be minimised. In this sense, such approach does maintain some of the qualities of other environments that employ LA such as Spotify or YouTube. Here the fixed dataset all users access is customised, re-arranged according to individual profiles, striking a balance that does not suppress either the granularity of the single or averages into a whole. The translation of these statistical patterns into spatial diagrams and layouts takes advantage of the granularity of data: it presents a high degree of diversification and relationality in order to absorb the discontinuities of the analytical process (Fig.3).

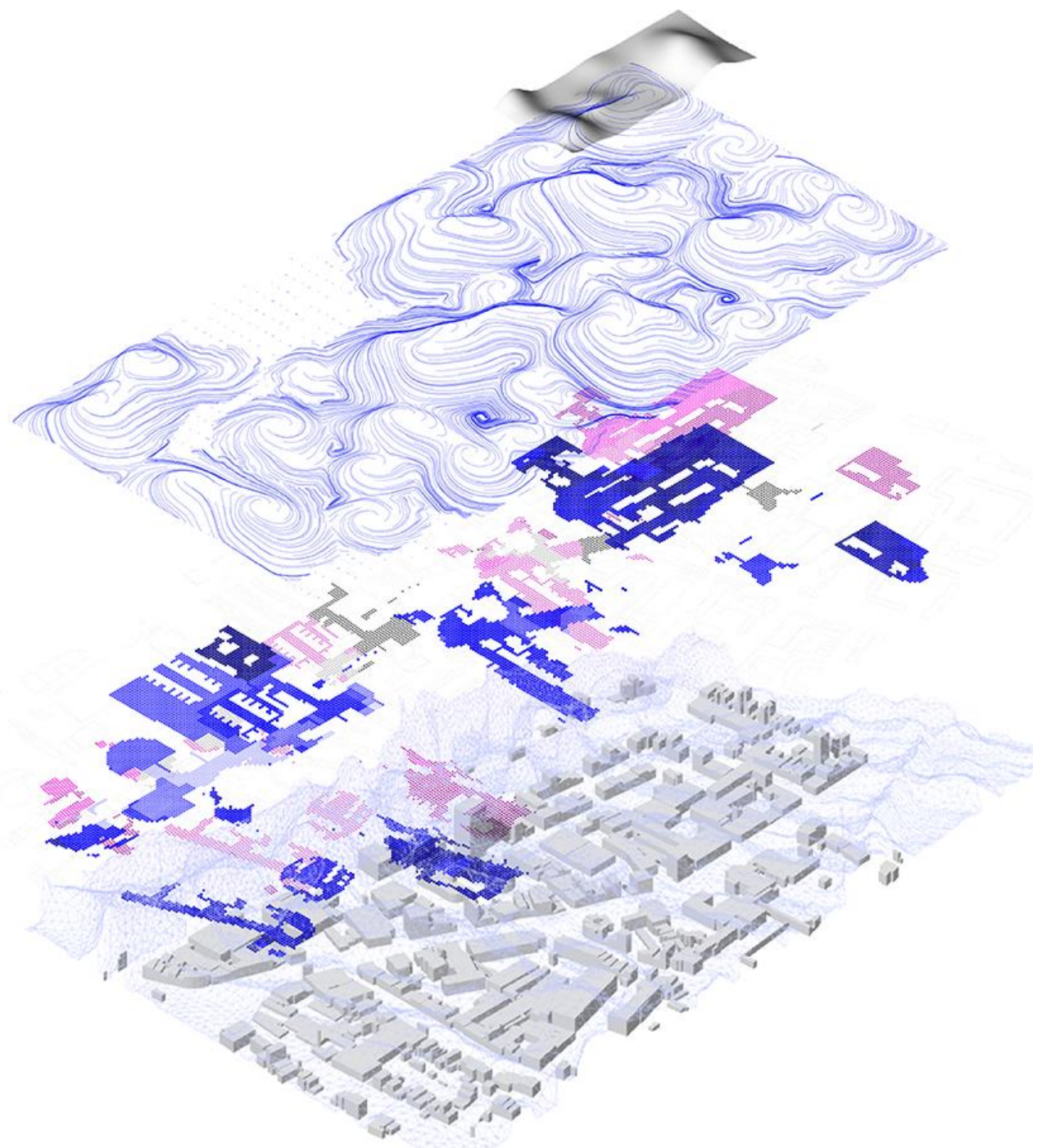

Figure 3: By comparing datasets in an axonometric drawing it possible to read the complex, discontinuous distribution of data typical in large data analysis. 
It is useful to recall Sini's discussion on prosthesis whose double job is to discriminate and measure the original set of information to which it was applied. LA perform this double task similarly to how orthographic drawings are used to present a piece of architecture: a plan or a section are literally singling out a precise set of information out the total dataset, whilst providing a set of instruments [e.g. planar projection, etc.] to remap and 'measure' the original dataset. The compound effect of these two operations is a different, constructed image of the original object, revealing invisible elements of the original dataset, and yet partial. Overseeing all these operations is the primary possibility to manage and mine datasets to re-write into forms as wholes, not necessarily having to move from the small to the larger.

The kind of spatiality implied by LA distance them from other processes to organise space employed by digital designers. Though they are often used in conjunction with parametric models (for instance, by combining them with a generative algorithm), LA are conceptually different. In fact, parametrics presupposes a certain process of idealisation which implicitly identifies an object or condition - no matter whether existing or not - from which to seed out a whole series of instances all related by continuous variation. This process leads to an idealisation of the computational model which goes beyond the inevitable approximation and reduction often accompanying the construction of a computational model. It implies a conceptual structure which ends up harmonising, equalising the actual distribution of values within the dataset. LA, on the other hand, allow to register a more detailed distribution of the data as they employ numerical methods operating through iterative approximations based on refinement, statistical distribution and correlation. Reduction towards some sort of idealised image is not part of their coding. Such approach provides a wider range of tools to work with numerical properties (clustering, distribution, discontinuities, etc. as shown in the examples of the t-SNE algorithm) as we move from algorithmic abstraction towards spatial organisation. Digital architects have long employed formulas such as "top-down" or "bottom-up" to explain their design process. The former often denoted more open, emergent qualities of design that were native to the digital. This characterisation
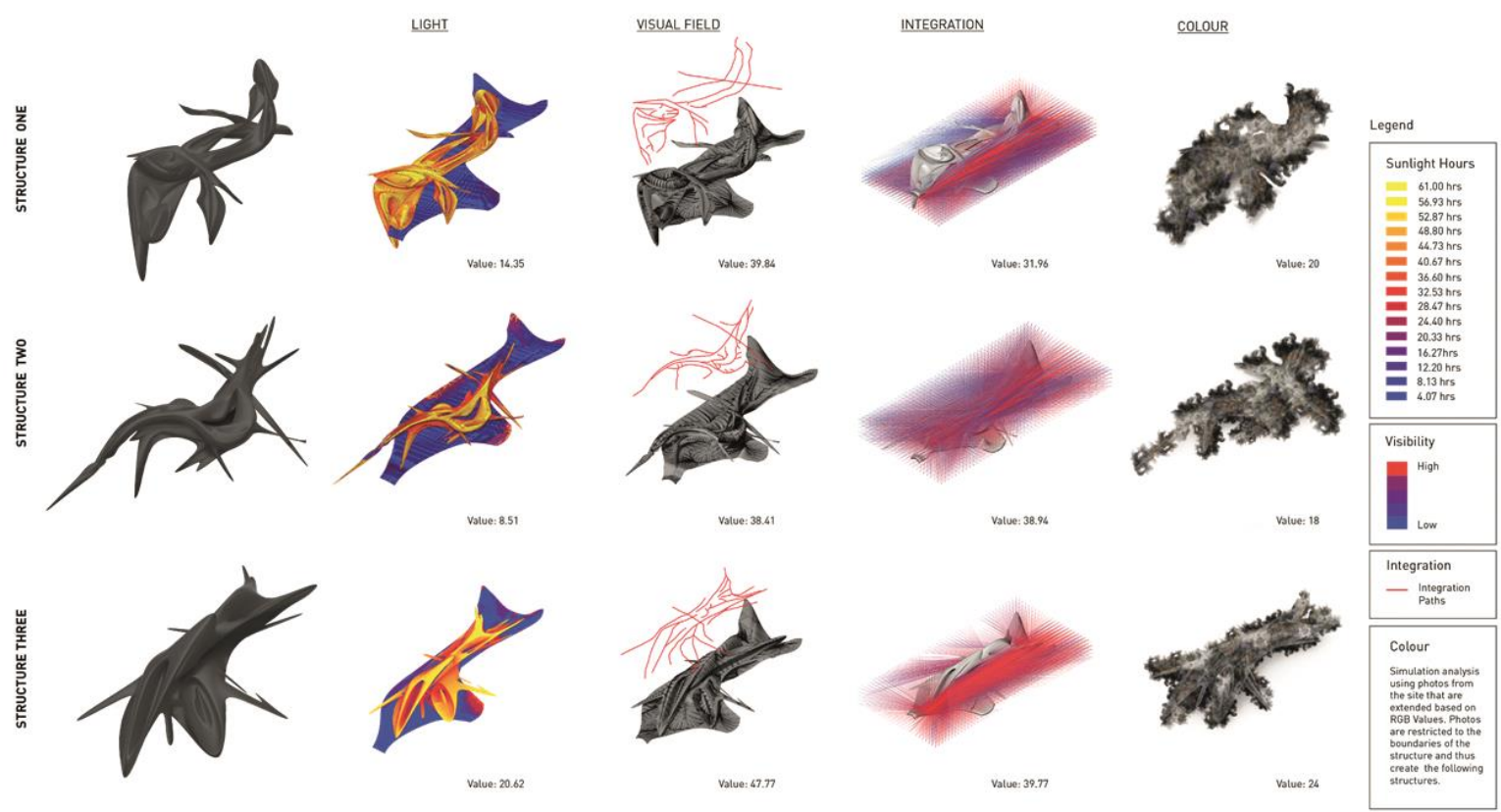

Figure 4: Diagrams showing the process of rewriting a particular dataset into a series of formal arrangements. The parameters of visual field, lighting and colour are analysed and used to construct new interpretations of the city. Machine learning algorithms, with linear data transformations, assist in revealing similarities and patterns within the data. 
of the work is often one that accompanies the use of parametrics in design. Parametric design implies a linear hierarchy in the organisation of the form: parameters vary gradually causing shapes to change in a continuous fashion. LA approach data by abstracting them from their actual geo-location and rearrange them in the abstract space of statistical distribution. A comparison between these two spaces (geo-located and abstract) reveals all sorts of jumps, voids, spatial inconsistencies. The morphological language able to capture such features cannot be that of continuous surfaces and incremental variation but rather a more fragmented one. Consequently, categories such as bottom-up and top-down lose their traction not so much because they do not apply anymore, but rather because they could both be utilised repeatedly and even simultaneously (Fig.4).

More interesting to deal with the new images produced by LA are operation of re-writing (another term borrowed from mathematics and linguistics) or, more aptly for design disciplines, nesting which can be interpreted both as generator morphological and programmatic organisations (Fig.5). In the field of mathematics, computer science and logic, rewriting procedures are understood as operations of "...replacing the subterms of a formula with other terms". ${ }^{9}$ By definition these operational are relational and transformative and, in mathematics, can take on non-deterministic rules of transformation. The application of such principles to morphological operations can be of great interest as the result may be able to absorb spatial and scalar inconsistencies whilst remaining logically coherent. Rewriting would no longer be relying on ideals situated in a transcendental domain but exploit patterns and randomness within the bounds of the dataset computed. For this reason, a more granular, fragments design language offers richer variety to rewrite the various datasets with more articulated and nested with more variations. This approach opens to a different aesthetics no longer seeking smooth and continuous morphologies (Fig.6). Some issues still demand further examination, such as how to relate the dynamism of data and the fundamental static nature of architecture, or how manage the

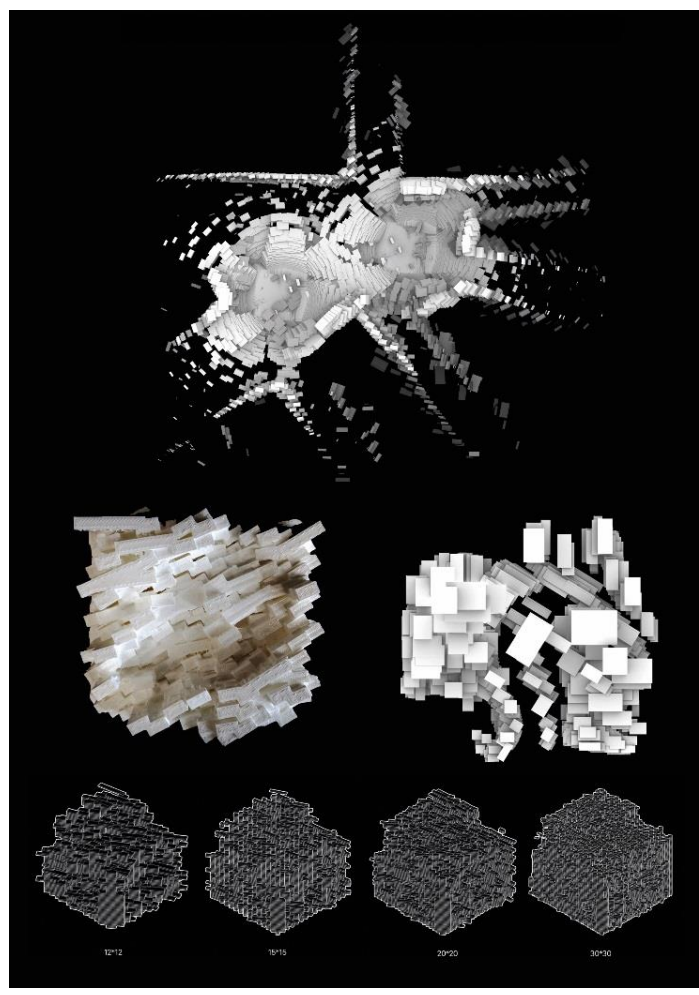

Figure 5: Examples of morphological re-writing based on different urban datasets concerning sound, geological conditions, and visual porosity.

potential data loss in in morphological rewriting a dataset.

\section{Conclusions}

This paper is as an initial attempt to contextualise and problematize the introduction of LA in design disciplines as well as show some applications developed within the academic context. The conflation of historical, technical, and disciplinary concerns and methods showed that the challenges posed by LA cannot be quickly limited to the simple improvement of traditional methods. Though the promise to make the design process more efficient is still a useful one, it cannot be the sole driver for design innovation. Beyond optimisation, the design

${ }^{9}$ Rewriting. Available from:

https://en.wikipedia.org/wiki/Rewriting [accessed on

October 29th, 2019]. 


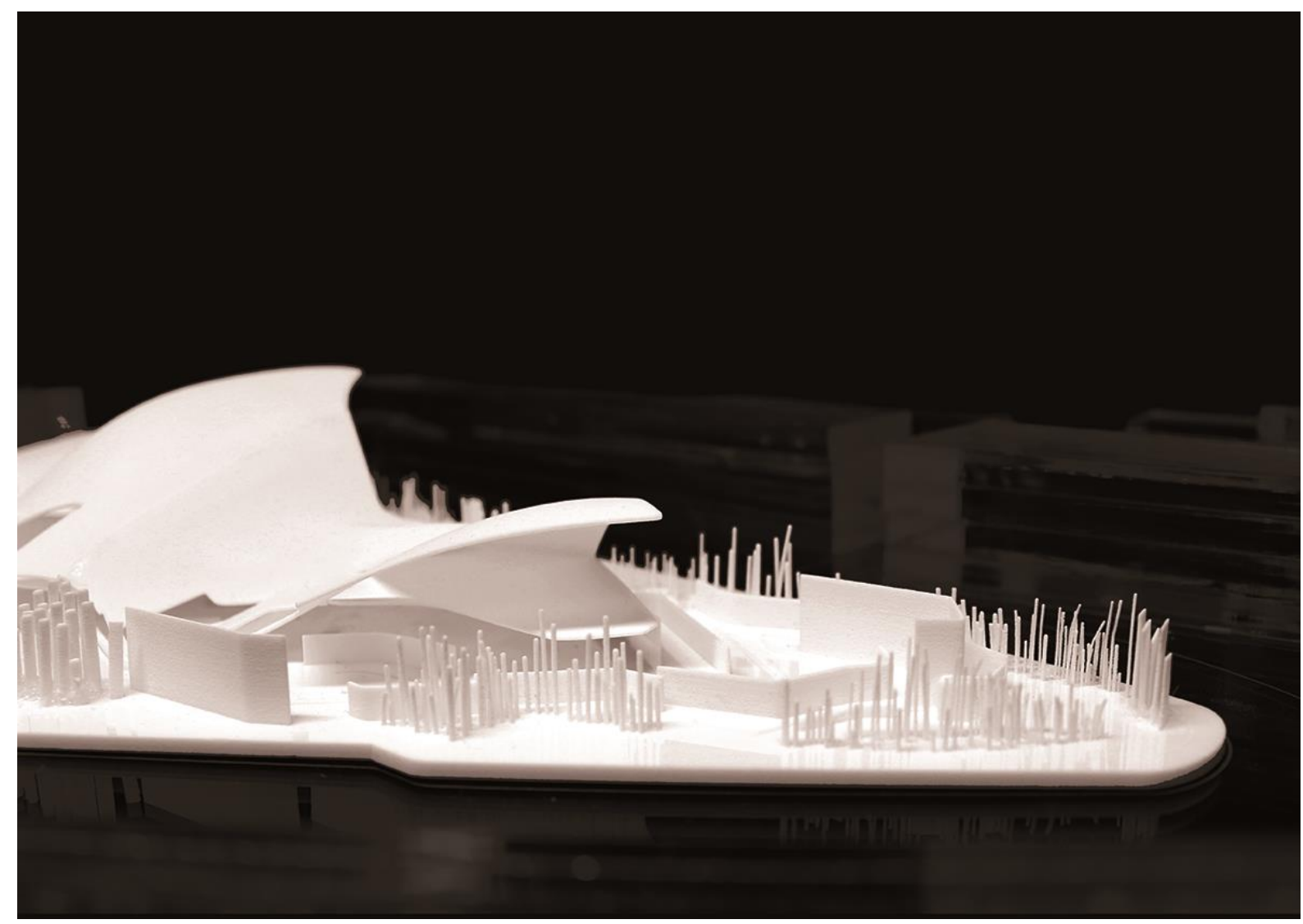

Figure 6: Physical models of one of the proposal developed by using learning algorithms. This particular project focuses on sound. By operating at the scale of the entire neighbourhood, this project imagines a series of small structures designed to either deflect, concentrate or altogether protect from sound. The centrepiece of this urban strategy is a large public space that can operate as a market, open-air auditorium, and public space.

instances discussed here show a more radical transformation at work. This shift not only concerns the very methods designers employ to "see" cities, but also the spatial models (both abstract and geographical) they could employ to organise their work. The discussion was keen to trace both the historical genealogy of this transformation as well as the points of rupture with the contemporary discourse on digital design. LA present both opportunities and challenges that will demand designers to be able to improve their digital literacy to grasp how space is computed by certain classes of algorithms and their agency on technological and disciplinary knowledge. In fact, the introduction of LA in design will unavoidably increase the automation of creativity and will shift designers' responsibility towards the understanding and shaping of systems more than individual objects. However, no design "revolution" can only focus on process without also altering outcomes. In this area, the use of discretised morphological language (a quantum approach) can be of help not only to absorb discontinuities, but also articulate the high-dimensionality of Big Data. On the one hand, issues of temporality, of conventions related to the formalisation of reality into data, represent some of the most pressing issues still in need of further research and refinement. On the other, the reward for working with LA will be to redefine aesthetic and performative qualities of architecture and urban design by going beyond our traditional understanding of concepts of scale, type, site and method. 


\section{References}

Baccaglioni, L., Del Canto, E. (1981). Leonardo Mosso: Architettura e Pensiero Logico. Mantua: Casa del Mantegna.

Bottazzi, R. (2018). Digital Architecture Beyond Computers: Fragments of a Cultural History of Computational Design. New York, London: Bloomsbury Academic. https://doi.org/10.5040/9781474258159

Coons, Steven A. (1966). "Computer, Art \& Architecture". In Art Education 19, no.5, May 1 1966, pp.9-11.

Hayles, K. (1993). Virtual Bodies and Flickering Signifiers. In October, Vol. 66, autumn issue, pp. 6991. https://doi.org/10.2307/778755

Link, D. (2016). Archaeology of Algorithmic Artefacts. Minneapolis: Univocal.

Manovich, L. (2013). Software Takes Command. New York, NY and London: Bloomsbury Academic.

McCarthy, J., Minsky, M., Rochester, N., Shannon, C.E., (1955). "A Proposal for the Dartmouth Summer Research Project on Artificial Intelligence". Available at:

http://raysolomonoff.com/dartmouth/boxa/dart564 props.pdf [Accessed on June 21, 2019].

Nietzsche, F. (1975). Frammenti Postumi 1885-1887, Italian translation by S. Giametta, Opere vol.8, Milan: Adelphi.

Sini, C. (2009). L'Uomo, La Macchina, L'Automa: Lavoro e conoscenza tra future prossimo e passato remoto. Turin: Bollati Boringhieri.

Turing, A. (1950). "Computing Machinery and Intelligence". In Mind, Volume LIX, Issue 236, October 1950, pp. 433-460.

https://doi.org/10.1093/mind/lix.236.433

\section{List of Figures:}

Figure 1

Credits: Vasileia Panagiotopoulou, Xinyi Li, and Ziyi Yang, Depraved urban spaces, B-Pro research Cluster 14, Bartlett School of Architecture, University College London (UCL), 2018.
Figure 2

Credits: Anna Kampani, Apostolopoulos Apostolos, and Caitlin Brock, Perceptive Datascapes, B-Pro research Cluster 14, Bartlett School of Architecture, University College London (UCL), 2018.

Figure 3

Credits: Anna Kampani, Apostolopoulos Apostolos, and Caitlin Brock, Perceptive Datascapes, B-Pro research Cluster 14, Bartlett School of Architecture, University College London (UCL), 2018.

Figure 4

Credits: Anna Kampani, Apostolopoulos Apostolos, and Caitlin Brock, Perceptive Datascapes, B-Pro research Cluster 14, Bartlett School of Architecture, University College London (UCL), 2018.

Figure 5

Credits: Mengyuan Li, Xingwen Qu, Tong Song, Xingyu Zhao (City Mountain team) and Nefeli Georgantzi, Shucheng Guo, Jiazhen Lu, Jingzhou Wang (LO-Fi team). B-Pro Research Cluster 14, Bartlett School of Architecture, University College London (UCL), 2019.

Figure 6

Credits: Guang Yang, Peng Zhou, Xiaoben Li, and Yu Han, Urban wave Decay, B-Pro Research Cluster 14, Bartlett School of Architecture, University College London (UCL), 2018. 